\title{
EL GIRO NEOLIBERAL DE LAS POLÍTICAS PARA LA CIUDAD EN ESPAÑA. BALANCE A PARTIR DE LOS EJEMPLOS DE MADRID Y VALENCIA
}

\author{
Juan Romero \\ Departamento de Geografía e Instituto Interuniversitario de Desarrollo Local. Universitat de València \\ juan.romero@uv.es \\ Dolores Brandis \\ Departamento de Geografía Humana. Universidad Complutense de Madrid \\ dbrandis@ghis.ucm.es \\ Carme Melo \\ Departamento de Geografía. Universitat de València \\ carme.melo@uv.es
}

\section{RESUMEN}

La globalización de la economía ha provocado grandes transformaciones en la nueva fase de desarrollo del capitalismo desregulado y sus consecuencias muestran su rostro más crudo en la profunda recesión posterior. El «giro neoliberal» de las políticas para la ciudad se concreta en España desde la segunda mitad de los noventa del siglo XX. En las páginas que siguen se analizan el modelo de urbanismo neoliberal, sus consecuencias en el territorio y los efectos de las políticas de austeridad en dos regiones urbanas y metropolitanas que representan la versión más acabada del modelo: Madrid y Valencia.

Palabras clave: ciudad neoliberal, desarrollo inmobiliario, gobierno de la ciudad, políticas de austeridad, Madrid, Valencia.

\section{ABSTRACT}

Economic globalisation has led to great transformations in the new phase of deregulated capitalism; the deep recession is one of its most dramatic consequences. In Spain, the

Fecha de recepción: noviembre 2013.

Fecha de aceptación: septiembre 2015. 
«neoliberal turn» in city policies initiated in the second half of the 1990s. This paper examines the neoliberal model of urbanism. A particular focus is placed on the territorial impact and the effects of austerity measures on two Spanish urban and metropolitan regions that represent the most elaborate version of the model: Madrid and Valencia.

Keywords: neoliberal city, real estate development, city government, austerity policies, Madrid, Valencia.

\section{INTRODUCCIÓN}

El rumbo trazado por la agenda neoliberal hace más de tres décadas además de ser hegemónico pretende ser irreversible. Las causas que explican lo que Krugman ha llamado «la gran divergencia» (2008) y Rosanvallon «el gran cambio» (2012) son de naturaleza política y responden a una estrategia que se remonta a los años setenta del siglo XX. El efecto combinado de la crisis de crecimiento y las políticas de la austeridad de inspiración neoliberal está modificando de forma profunda nuestras estructuras sociales desde la conocida forma de rombo hacia estructuras sociales mucho más complejas y fracturadas (Savage et al. 2013) donde se han consolidado nuevas formas de inseguridad (Standing, 2013). Es el resultado de lo que Beck ha definido como «la política económica de la inseguridad»: incremento de la pobreza y la desigualdad, precarización del empleo e inseguridad endémica, traslado de los riesgos al individuo, adelgazamiento de las clases medias, aumento de las fracturas sociales, ruptura del vínculo existente entre capitalismo, Estado de Bienestar y democracia, riesgo de brasilización de occidente, aumento de la desafección y polarización social (2012). Por ello, la Cuestión Social debe ser motivo de atención creciente para las ciencias sociales (Romero, 2013).

Las consecuencias de estas transformaciones son tremendamente visibles en las ciudades. En las páginas que siguen se analiza el modelo de urbanismo neoliberal y los efectos de las políticas de austeridad en dos regiones urbanas y metropolitanas españolas en las que durante más de dos décadas los gobiernos conservadores han sido hegemónicos tanto en la escala local como regional: Madrid y Valencia. Suele argumentarse que frente al «modelo» de ciudad neoliberal «puro» de Madrid y Valencia existen otras versiones soft de urbanismo neoliberal. Los casos de Barcelona y Bilbao serían buenos ejemplos (Cucó, 2013b; Capel, 2005; Delgado, 2007; Borja, 2010; Esteban, 2008). Si bien es cierto que otras ciudades y regiones urbanas y metropolitanas españolas han ensayado las mismas políticas (VVAA, 2013), parece existir amplia coincidencia en señalar que Madrid y Valencia serían casos paradigmáticos de aplicación del pensamiento neoliberal en el gobierno de la ciudad (Rodríguez et al, 2013; Cucó, 2013a).

\section{EL MODELO NEOLIBERAL DE PRODUCCIÓN Y REPRODUCCIÓN DE CIUDAD}

El «giro neoliberal» de las políticas para la ciudad se concreta en España desde la segunda mitad de los noventa del siglo XX. Éste se ha plasmado, como señala Jordi Borja, «en la arquitectura de autor, la oferta de áreas para la nueva economía, la gentrificación (o la 
museificación) de la ciudad consolidada, la mercantilización del valor simbólico del patrimonio, el miedo justificador de los barrios cerrados, el crecimiento periférico por piezas y funciones especializadas...» (2013:30).

Partiendo de esta definición podrían añadirse algunas características básicas: a) una apuesta clara por la producción inmobiliaria como motor de la actividad económica; b) cambios importantes en la legislación urbanística que favorecieron la liberalización y revalorización del suelo, la urbanización masiva y dispersa en el territorio de grandes desarrollos residenciales fuera de los planes urbanos y la apropiación de la renta por parte de promotores y entidades financieras; c) cambios en la legislación hipotecaria que propiciaron la financiación del suelo y su integración en los circuitos globales de capital a través de la titulización hipotecaria; d) una apuesta decidida a favor del llamado «urbanismo de promotor» o de «emprendedor» de la mano de sólidas coaliciones entre entidades financieras, promotores y autoridades locales y regionales (Harvey, 2012); e) atención preferente a la promoción de vivienda libre en detrimento de las políticas públicas de fomento de vivienda protegida; f) enormes inversiones públicas en Grandes Eventos y Grandes Proyectos singulares, con su complemento imprescindible de grandes infraestructuras (aeropuertos, ferrocarril, puertos, autovías); g) privatización del espacio y de servicios públicos (Díaz, 2013; Vives y Rullán, 2014); h) desarrollo de una concepción peculiar del ideario neoliberal consistente en la firme defensa del retroceso del Estado en todo lo relacionado con fiscalidad progresiva y provisión de bienes y servicios públicos y al tiempo utilizar el Estado para la obtención de beneficios privados.

Estos rasgos son el resultado de un amplio conjunto de políticas que se aleja del tantas veces reivindicado derecho a la ciudad y del buen gobierno del territorio en las regiones metropolitanas, que no concibe el urbanismo como instrumento político, capaz de prever el crecimiento económico y demográfico de la ciudad, y de programar la urbanización y equipamiento para canalizar sus efectos espaciales (Pinson, 2011), sino como la búsqueda del crecimiento a toda costa y la privatización de servicios y del espacio público (Méndez, 2012).

Este modelo, bien explicado por distintos autores (Naredo, 2010; Gaja, 2013; Sorribes, 2015), se apuntala cediendo de facto las responsabilidades del desarrollo y ordenación territorial a los gobiernos locales que, a su vez, delegan en las elites la definición y ejecución del desarrollo urbano. Se trata, pues, de acabar con el planeamiento como instrumento fundamental para ordenar y construir de manera racional la ciudad, para programar la distribución óptima de personas, bienes, equipamientos y servicios sobre el territorio y para imponer a los agentes privados una disciplina urbanística. Es así como el planeamiento deja de ser una garantía de racionalidad territorial y de defensa del interés general, para convertirse en un instrumento de seguridad jurídica al servicio de intereses económicos particulares.

Sobre el terreno, esta forma de concebir el urbanismo contempla, por un lado, acondicionar la ciudad consolidada mediante transformaciones urbanas de reproducción de determinados espacios, concebidos dentro de una planificación estratégica y ejecutada mediante proyectos singulares y arquitecturas de autor, que responden a los retos de la globalización y de la competitividad interurbana internacional. En este sentido, Madrid cuenta con los ejemplos emblemáticos del complejo «Cuatro Torres Business Area», que ha dado lugar al nuevo skyline de la ciudad, y la «Operación Chamartín», bloqueada por la crisis inmobiliaria. El caso de Valencia es, si cabe, más espectacular y dramático. La gran apuesta de los 
responsables políticos por Grandes Proyectos y eventos deportivos (Ciudad de las Artes y las Ciencias, Copa de la América y circuito de Formula 1) ha supuesto un formidable despilfarro de recursos públicos en proyectos ruinosos y finalmente fracasados, como explicaremos.

A las políticas de inspiración neoliberal también corresponden las propuestas de crecimiento urbano en las periferias de las ciudades, valorizadas como potencialmente urbanizables. El territorio se concibe como un simple soporte para dinamizar la actividad económica, y donde los agentes privados obtienen inmensas plusvalías derivadas de su renta por la reclasificación de terrenos, su urbanización y construcción. Y así, uno de los pilares para llevar a cabo las políticas neoliberales ha sido la liberalización del suelo, esto es, la declaración de todo el suelo no protegido como urbanizable. Madrid constituye, como argumentaremos, el mejor ejemplo de este modelo con las desproporcionadas operaciones residenciales de los Programas de Actuación Urbanística (PAU) de 1991 y de los desarrollos de 1997, que han supuesto la liberalización de ocho mil hectáreas de suelo. El caso de Valencia, por su parte, refleja el fracaso de formas de gobierno metropolitano, destrucción progresiva de uno de los paisajes culturales más importantes de la cuenca del mediterráneo, la Huerta periurbana de Valencia, por el avance urbanizador y el trazado de nuevas infraestructuras (Zornoza, 2013; Burriel, 2014).

Este proceso ha sido fruto de una estrategia concertada (sistema financiero, promotores y representantes políticos) en un contexto desregulado y sin controles que favoreció la formación de una extraordinaria burbuja inmobiliaria especulativa (Fernández, 2008; Burriel, 2008; 2014; Campos, 2008; Naredo, 2010; Naredo y Montiel, 2011; Romero et al, 2012; Vinuesa, 2013). Una situación que priorizaba la construcción nueva frente a la rehabilitación, la vivienda como inversión frente a la vivienda como bien de uso, la vivienda libre frente a la vivienda social, la vivienda en propiedad frente a la vivienda en alquiler y la rentabilización a través de plusvalías.

A continuación se abordan con más detalle las consecuencias de la política urbana neoliberal en dos regiones urbanas y metropolitanas españolas que representan dos ejemplos del mismo proceso y la versión más acabada del modelo: Madrid y Valencia.

\section{EL APOYO DE LAS ADMINISTRACIONES PÚBLICAS AL DESARROLLO INMOBILIARIO DE MADRID}

La aplicación del modelo de urbanismo neoliberal, así como sus consecuencias, se reconocen, a distintas escalas, en el proceso de producción seguido en muchos territorios del país y, entre ellos, la periferia madrileña. Se trata de una producción de periferia residencial, porque la enorme cantidad de suelo urbanizable puesto en el mercado entre 1991 y 1997 en el municipio de Madrid (8.000 hectáreas) estaba destinada a la edificación de viviendas (más de 200.000 previstas) (Brandis y Río, 2007; Brandis, 2012a; 2012b).

El gobierno municipal conservador de 1991 acometió la revisión del planeamiento para establecer una nueva filosofía urbanística, contraria a la idea de contención que achacaba como defecto al Plan General de 1985 y que impedía dinamizar la economía regional mediante la urbanización y la construcción. La estrategia consistía en movilizar rápidamente suficiente suelo donde construir una gran cantidad de viviendas, argumentando que la escasez de suelo había generado una espiral especulativa, provocando la expulsión de los madri- 
leños hacia la periferia metropolitana por la escasez y carestía de la vivienda. Y es así como se pone en marcha la que será la mayor intervención urbanística de la historia al margen de lo planeado (Brandis, 2014).

El proceso contó con el apoyo directo del gobierno conservador regional cuyo Plan Regional de Estrategia Territorial de 1996 contribuyó a la urbanización de forma activa y la impulsó mediante fuertes inversiones en infraestructuras por el territorio metropolitano, que vinieron a complementar las realizadas por el gobierno central. En paralelo, la mayoría de los gobiernos locales de la Comunidad Autónoma aprobaron planeamientos urbanísticos para extender notablemente la superficie urbanizable y favorecer la actividad constructora, fundamental para aumentar sus ingresos.

Los mismos presupuestos de crecimiento del parque inmobiliario subyacen en el Plan de Ordenación de Madrid aprobado en 1997 por el gobierno conservador, que apuesta por el urbanismo de expansión iniciado en 1991. Se procedió a una reclasificación masiva de suelo no urbanizable protegido en el plan de 1985. Allí se pretendía levantar en cinco mil hectáreas más de 100 mil viviendas en siete nuevas macro actuaciones.

Para facilitar el crecimiento inmobiliario de los PAU de 1991 y de los posteriores desarrollos urbanísticos de 1997 era imprescindible institucionalizar la práctica negociadora para facilitar a los agentes económicos intervenir con seguridad. Se procedió a agilizar la gestión, con el fin de reducir el tiempo que media entre la redacción del planeamiento y la construcción de las viviendas, así como para limitar la acción de la administración en la definición de las directrices del planeamiento, fijando unas horquillas de edificabilidad y dando a los propietarios una gran libertad para el diseño de los planes parciales. Las grandes empresas constructoras e inmobiliarias se constituyeron en los principales agentes urbanizadores que movilizaron grandes cantidades de suelo para construir viviendas, apoyadas en el sector financiero que aportó el crédito barato necesario para alimentar la burbuja. Este mercado, de rápidas venta sobre plano y subidas incesantes del precio de las viviendas, entró en crisis de forma abrupta en 2007.

El simplismo y la falta de complejidad de las formas urbanas han sido la norma en los proyectos de crecimiento residencial, dando lugar a un conglomerado estereotipado que permite situarlos en cualquier parte del territorio. La insularidad es una de las características, y su situación al borde de autopistas dificulta sobremanera las conexiones con las tramas urbanas próximas. Las reducidas densidades residenciales, la falta de integración de usos y el sobredimensionamiento de un viario compuesto por vías transversales de gran sección, absolutamente disuasorias para el tránsito peatonal, hacen de estos PAU banales ejercicios de composición urbana. La ausencia de espacios públicos como lugar de encuentro se palia, en parte, con las dotaciones privadas en el interior de los edificios residenciales. Se trata de un diseño cerrado al exterior por muros y verjas, que elimina toda posibilidad de introducir comercios a pie de calle, auténticos dinamizadores tradicionales de la vida callejera. Además, el modelo seguido conlleva la introducción de un nuevo componente, el gran centro comercial, que se constituye en el espacio público por excelencia en la concepción neoliberal de ciudad y parte indiscutible de la ordenación.

El proceso de ejecución de los ámbitos proyectados se ha visto frustrado por la crisis económica. Ni han culminado todos los PAU, ni apenas han empezado a ver la luz los últimos desarrollos. En las ocho mil hectáreas de periferia propuestas para urbanizar a partir de la 
última década del siglo pasado, donde se preveía construir más de 200 mil viviendas, existen actualmente menos de 80 mil, el $38 \%$, pues hay edificios sin terminar y otros sin iniciar todavía (Brandis, 2014).

El principal problema de la urbanización de la periferia no ha sido la escasez de suelo urbanizable y de viviendas, sino su exceso e infrautilización, y la ausencia de voluntad de los actores concernidos para imaginar políticas públicas coherentes, equilibradas y sostenibles. El marco institucional hizo del negocio constructivo-inmobiliario la verdadera industria nacional y desencadenó un tsunami de obras que, además de impactar negativamente sobre la calidad de vida, el patrimonio urbano y los ecosistemas, originó viviendas desocupadas y necesidades insatisfechas (Naredo, 2009).

En resumen, un ciclo inmobiliario que alcanzó dimensiones espectaculares como se desprende del trabajo hecho público desde el Observatorio Metropolitano de Madrid: entre 1993 y 2003 se comprometieron 50.000 hectáreas para desarrollos inmobiliarios en la Comunidad de Madrid. Los nuevos barrios planearon 180.000 nuevas viviendas al tiempo que en la Comunidad se construyeron cerca de 800.000 viviendas entre 1995 y 2008 (Rodríguez et al, 2013).

\section{EL FRACASO DEL MODELO DE «CIUDAD ESPECTACULARIZADA» Y DE GOBIERNO DEL ÁREA METROPOLITANA EN VALENCIA}

El ejemplo de Valencia es, en lo básico, similar, por cuanto se han desarrollado políticas de idéntica orientación. Sin embargo, no estuvo tan centrado en la urbanización masiva del área metropolitana sino en grandes desarrollos inmobiliarios, en la promoción de mega eventos y en la inversión en grandes proyectos singulares para la ciudad mediante Planes Parciales y Planes de Actuación Integrada aprobados ad hoc. Proyectos o eventos singulares que en ocasiones se han impulsado en detrimento de políticas urbanas para toda la ciudad, incrementando la percepción entre los ciudadanos de que existía una ciudad para ser visitada y otra ciudad, no tan glamurosa, ni siquiera bien atendida por los poderes públicos, para residir. El intento fracasado de consolidar un circuito permanente de Fórmula 1 es un buen ejemplo de esa ideología de lo efímero, de la ultramodernidad, que fundamentó el proyecto de la Valencia neoliberal de las dos últimas décadas (Ruiz y Santamarina, 2013).

Fernando Gaja ha elaborado un excelente balance de este modelo urbanístico impulsado por responsables políticos locales y regionales, que a falta de «una idea global de ciudad a medio y largo plazo» apostaron por la «celebración de Grandes Eventos, vinculándolos al desarrollo de Grandes Proyectos Urbanos, una opción opaca, cara, inequitativa, improvisada y derrochadora» (Gaja, 2013:214-215). Sirva como ejemplo de despilfarro (no exento de episodios de corrupción pública) el coste estimado acumulado de la inversión pública de los proyectos más emblemáticos solo en la ciudad de Valencia: America's Cup (2.064 millones de euros); Ciudad de las Artes y las Ciencias (sobre 1.300 millones de euros) y circuito de Formula 1 (en torno a 235 millones de euros).

El fracaso del proyecto de «ciudad neoliberal espectacularizada» (Hernández y Torres, 2013) deja ahora importantes consecuencias en la ciudad «ocultada» (Torres y García, 2013): privatización del espacio urbano, aumento de la deuda pública municipal y regional, relegación material y simbólica de muchos barrios y numerosos edificios e infraestructuras inaca- 
badas, abandonadas o infrautilizadas. El contraste viene definido por la retirada del gobierno local en la prestación de servicios públicos y por el aumento de la fragmentación, la desigualdad y la precariedad.

Hoy ya es evidente que este modelo ha conducido a la ciudad de Valencia y al conjunto de la región a una situación muy difícil. El ayuntamiento de Valencia se encuentra ahora en una situación económica muy precaria con un nivel de endeudamiento público (875 millones de euros en enero de 2014) que reduce de forma drástica las posibilidades de un buen gobierno para la ciudad. La región metropolitana de Valencia, además de afrontar idénticos desafíos relacionados con el gobierno de la ciudad «real», cuenta con una de las cinco grandes Huertas de todo el mediterráneo occidental (Stanners y Bourdeau, 1995; Romero y Francés, 2012). Pocas ciudades europeas disponen de entornos tan singulares y valiosos. No obstante, los actores políticos han sido incapaces de liderar e impulsar políticas coherentes para garantizar una ordenación y gestión sostenibles (Romero y Farinós, 2013).

La Huerta de Valencia ha participado de los mismos procesos de desgobierno metropolitano ocurrido en las grandes áreas metropolitanas españolas durante los últimos años: expansión caótica y masiva del urbanismo residencial, localización desordenada de actividades industriales y de servicios, especulación del suelo y degradación medioambiental. Estas son las consecuencias visibles de la ausencia de planificación a escala metropolitana, de la incuria e inhibición de las administraciones autonómicas concernidas, que haciendo dejación de sus competencias en materia de ordenación del territorio no han querido establecer marcos de ordenación y gestión a escala supralocal, de la voracidad de decenas de corporaciones locales que no han reparado en las consecuencias irreversibles del proceso y de la ausencia de una apuesta decidida por hacer de los agricultores pieza fundamental de un modelo de producción y consumo distinto. El proceso inacabado del Plan de Acción Territorial de Protección de la Huerta de Valencia (inicialmente sometido a información pública en 2010 y más tarde abandonado por el gobierno regional), ha sido el último episodio de un fracaso en el que la obstrucción política del ayuntamiento de Valencia fue decisiva (Romero y Melo, 2015).

\section{POLITICA DE AUSTERIDAD, RECORTES DE GASTO PÚBLICO SOCIAL Y PRIVATIZACIO- NES: CONSECUENCIAS EN LAS CIUDADES}

La política de austeridad y de desguace del Estado de Bienestar impulsada en España por los gobiernos conservadores obedece, a nuestro juicio, a motivaciones políticas y no económicas. La agenda neoliberal es clara: reducción lineal del gasto público social y privatización de servicios, frente a otras visiones que enfatizan que reforma no significa únicamente recorte de gasto público social sino que es posible incorporar mejoras en la eficacia y la eficiencia que hagan sostenible el Estado de Bienestar, garantizando servicios universales y públicos (Romero et al, 2014).

La Actualización del Programa de Estabilidad del Reino de España (Ministerio de Hacienda, 2013) prevé reducir el peso del gasto en el PIB desde un 45,2\% de 2010 al 39,7\% en 2017. Ello situará a España casi un $25 \%$ por debajo de la media europea en tamaño relativo del sector público y, en consecuencia, en dimensión y prestaciones de su Estado de Bienestar (Tabla 1). 
Tabla 1

DESGLOSE DE FUNCIONES DE GASTO. PROGRAMA DE ESTABILIDAD DEL REINO DE ESPAÑA (2011-2016)

\begin{tabular}{lccc}
\hline Funciones de gasto público & $\begin{array}{c}2011 \\
(\% \text { PIB })\end{array}$ & $\begin{array}{c}2016 \\
\% \text { PIB })\end{array}$ & $\begin{array}{c}\text { \% Variación del peso } \\
\text { del gasto en el PIB }\end{array}$ \\
\hline 1 Servicios públicos generales & 5,7 & 6,1 & 7,2 \\
\hline 2 Defensa & 1,1 & 0,8 & $-20,6$ \\
\hline 3 Orden público y seguridad & 2,2 & 1,7 & $-22,4$ \\
\hline 4 Asuntos económicos & 5,3 & 3,3 & $-38,0$ \\
\hline 5 Protección del medio ambiente & 0,9 & 0,6 & $-30,6$ \\
\hline 6 Vivienda y servicios comunitarios & 0,6 & 0,4 & $-42,4$ \\
\hline 7 Salud & 6,3 & 5,4 & $-15,6$ \\
\hline 8 Actividad recreativas, cultura y religión & 1,5 & 1,0 & $-36,1$ \\
\hline 9 Educación & 4,7 & 4,0 & $-15,7$ \\
\hline 10 Protección social & 16,9 & 16,6 & $-1,8$ \\
\hline Gasto total & 45,2 & 39,7 & $-12,0$ \\
\hline
\end{tabular}

Fuente: Ministerio de Hacienda y Administraciones Públicas. Actualización del Programa de Estabilidad del Reino de España 2013-2016, 26 de abril de 2013.

La mayor parte de los recortes en políticas sociales corresponde a las Comunidades Autónomas y a los gobiernos locales, que son los niveles de gobierno responsables de la mayor parte de las políticas públicas sociales, al tiempo que el gobierno central, partiendo de una interpretación tan equivocada como discutible, les exige mayor porcentaje de reducción del déficit público. Como es en las ciudades donde se acaban concretando esas políticas, es lógico que sea en las ciudades donde las consecuencias son más visibles. Madrid y Valencia han sido también escenario de los efectos del despliegue de políticas muy orientadas a reducir el gasto público social y a privatizar las partes del Estado de Bienestar que son negocio. Entre los más destacables, nos centraremos en el incremento de la pobreza y la desigualdad social, el aumento de la segregación educativa, los desequilibrios en el derecho a la salud y, como reacción, la movilización política de amplios sectores ciudadanos.

\section{V.1. Aumento de la pobreza y desigualdad social}

La recesión ha ocasionado en España desde 2008 un aumento muy notable de la pobreza con niveles alarmantes en algunas Comunidades Autónomas (Herrero et al, 2012) y en determinados grupos de población. A ello se añade «un aumento de la desigualdad sensiblemente superior al registrado en promedio por los países de la Unión Europea» (Ayala et al, 2013: 52). Esta afirmación viene precedida por cuatro evidencias empíricas que hacen de España un caso singular: a) la crisis ha tenido un efecto negativo sobre el empleo muy superior al promedio; b) se ha producido el mayor aumento de la desigualdad desde que se dispone de información anual sobre los ingresos de los hogares; c) los hogares con menos ingresos son los que más han reducido sus recursos a diferencia de los hoga- 
res con mayores ingresos que han reducido menos sus ingresos, y d) España no solo ha demostrado una limitada capacidad para combatir el aumento de las desigualdades a través del sistema de impuestos y prestaciones, sino que los procesos de empobrecimiento económico y movilidad social descendente podrían verse agravados por los recortes en materia de protección social, afectando tanto a los ingresos como a la igualdad de oportunidades, incidiendo muy especialmente en los grupos más vulnerables, en especial jóvenes, personas migrantes, mujeres y parados de larga duración (Davia, 2013; Gradín y Del Río, 2013; FOESSA, 2014; Schraad-Tischler, Kroll, 2014).

También es importante el desigual impacto territorial. La crisis del sector inmobiliario ha afectado de modo más intenso a aquellas regiones en las que el avance del proceso urbanizador había generado mayor actividad y empleo asociado a la construcción. En consecuencia, el masivo y brusco aumento del desempleo, la pobreza urbana y la exclusión social registran niveles más elevados en determinadas regiones urbanas y metropolitanas, como Madrid, y en espacios litorales, muy especialmente en la región de Valencia, donde los indicadores de paro han empeorado de forma acelerada a partir de 2008 (Méndez et al, 2015; Pitarch, 2014; Salom y Albertos, 2014).

El Avance del VII informe de exclusión 2014 de la Fundación Foessa, hecho público por representantes de Cáritas en junio de 2014, indica que el 65\% de los hogares valencianos (495.000) se encuentra ya afectado por procesos de exclusión. El 11,5\% de las familias (218.000) sufre pobreza severa (un 2,7\% más que la media española) y solo el $35 \%$ goza de plena integración. Un proceso similar de incesante polarización, precariedad y fragmentación social, de crecientes dificultades para el proletariado de servicios y de transición hacia estructuras sociales muy fragmentadas se produce en la ciudad de Madrid (Rodríguez et al, 2013).

Especial atención merecen la infancia y los jóvenes a la vista de los análisis recientes (UNICEF, 2014; Save the Children, 2013). Los rasgos que mejor resumen la situación en la que se encuentra la mayor parte de la población menor de 29 años son desempleo, pobreza, precariedad, vulnerabilidad y escasas expectativas de movilidad social en el futuro. Dos datos son suficientes para resumir la situación: el 80\% de los 3,5 millones de puestos de trabajo destruidos en España desde 2007 corresponden a menores de 29 años y el porcentaje de jóvenes que no estudian ni traban (un 24,4\%) es una de las cifras más altas de todos los países desarrollados (Castelló, 2013).

\section{V.2. Desigualdad y segregación educativa: la gran fractura social}

Informes recientes sitúan a España en el último lugar de la Europa de los 28 en el campo de la educación y relacionan el dato con las políticas de austeridad (Schraad-Tischler y Kroll, 2014). Cuando se necesitan más recursos en formación y un programa realista de reformas del sistema educativo español y de los subsistemas de formación y aprendizaje, se entiende muy mal el compromiso adoptado con Bruselas por el actual gobierno español y su exigencia para que los gobiernos regionales hagan lo propio: reducir un $15,7 \%$ del gasto público en educación hasta 2017, pasando del 4,7 al 4,0 del PIB. Tal es la cifra que ofrece la Actualización del Programa de Estabilidad del Reino de España solo para el periodo 2013-2016, si bien los recortes serán muy superiores si se considera desde 2007. 
Medidas como la supresión masiva de profesores, las jubilaciones forzosas, la amortización de plazas de profesores interinos, la prohibición de sustituir a profesores jubilados o la congelación de sistemas de promoción a la docencia, el incremento del número de alumnos por aula, el aumento de las tasas académicas al tiempo que se introducen recortes lineales en materia de ayudas y becas al estudio, siguiendo el «modelo anglosajón» ultraliberal en vez del modelo continental (CRUE, 2015), la reducción drástica de los presupuestos a los centros públicos y a las universidades, o la jibarización del sistema público de ciencia y tecnología, tendrán repercusiones tan negativas como duraderas para el conjunto de la sociedad española.

De nuevo Madrid y Valencia han liderado la «revolución conservadora» en educación en España (Játiva, 2013). Pero como ocurre en sanidad, no solo actúa en el plano de los recortes lineales de gasto público reduciendo las oportunidades para la igualdad, sino que durante décadas los gobiernos conservadores de Madrid y Valencia han desarrollado su propia agenda con el objetivo estratégico de disminuir de forma gradual el peso de la educación pública en favor de la educación concertada y privada. Esa prioridad nada tenía que ver con la recesión sino con la ideología.

\section{V.3. Desequilibrios en el derecho a la salud}

El gobierno español se ha comprometido a reducir el gasto público en sanidad un 15,6\% entre 2011 y 2017, pasando del 6,3 al 5,4 del PIB. Eso supone una reducción cercana a los 10.000 millones de euros en un sistema sobre el que los recortes son mayores si se considera el periodo 2007-2017 y que había alcanzado un buen nivel de prestaciones. En paralelo, y por razones que no responden a una gestión más eficiente de recursos sino a motivaciones ideológicas, hace años (mucho antes de que la recesión fuera una realidad) que el partido conservador impulsa procesos de privatización del sistema público de salud en algunas Comunidades Autónomas.

La Comunidad Valenciana fue pionera en introducir formas de gestión privada en hospitales de titularidad pública y en «externalizar» distintos servicios. Un modelo que a partir del Plan de Infraestrucuras Sanitarias 2004-2007 se incorporó a la Comunidad de Madrid a través de ocho nuevos hospitales de titularidad pública, pero cuya construcción, mantenimiento y funcionamiento se adjudica a empresas privadas por un periodo de tiempo de hasta treinta años a cambio de un canon anual. (Rodríguez et al, 2013). Ya en recesión, la Comunidad de Madrid ha intentado entregar varios hospitales de la red regional, prácticamente en régimen de oligopolio, a grupos inversores integrados por bancos, empresas de servicios y fondos de inversión nacionales y extranjeros (proceso frustrado en parte gracias a la reacción de los movimientos sociales). A esto se añade el gran retroceso experimentado en los derechos a las personas en situación de dependencia y de sus familias a partir de la entrada en vigor del Real Decreto-Ley 20/12, que de hecho supone la práctica destrucción de un pilar fundamental de los Servicios Sociales en gran medida complementario al sistema de salud (Observatorio de la Dependencia, 2013; Asociación Estatal de Directoras y Gerentes en Servicios Sociales, 2015).

Los recortes en sanidad están ocasionando un apreciable deterioro de un sistema público de salud que se encontraba entre los diez mejores del mundo. Así es percibido con creciente 
preocupación por una mayoría de ciudadanos que siguen siendo partidarios de la sanidad pública como se indica en el Barómetro Sanitario difundido en agosto de 2013 (CIS- Ministerio de Sanidad, Servicios Sociales e Igualdad, 2012).

De otra parte, y en paralelo, se asiste a un creciente aumento de la contratación de servicios sanitarios privados por parte de segmentos de población con suficiente nivel de renta. En definitiva, deterioro y saturación de la red pública, privatización de parte de la misma o de parcelas muy rentables y desarrollo creciente de la red privada paralela. Un modelo que deja de equipararse al modelo tradicional imperante en Europa occidental para deslizarse de forma silenciosa pero irreversible hacia el modelo anglosajón.

\section{V.4. Desafección política y nuevos movimientos sociales}

La crisis gradual de los grandes partidos tradicionales y la emergencia de nuevos movimientos sociales urbanos que han ido apareciendo en los años de la crisis, desde el movimiento de «los indignados» y las masivas movilizaciones ciudadanas en favor de la sanidad y la educación públicas, las conocidas mareas ciudadanas especialmente importantes en Madrid, hasta las plataformas en defensa de afectados por los desahucios o los salvemos (El Cabanyal, el Botánico, la Punta o la Huerta en el caso de Valencia), expresan en parte lo que hay de verdaderamente nuevo en el actual contexto. Porque remiten a otra forma de ver la realidad, de entender la democracia, la política y el compromiso cívico. También de entender la ciudad como espacio político.

Se trata de movimientos que tienen una indudable dimensión política (Castells, 2011). No tienen nada que ver con el mayo del 68, no son movimientos antisistema, sino todo lo contrario, tampoco son sólo movimientos de jóvenes, y como ha quedado evidenciado no se trataba de movimientos efímeros. En los países de Europa meridional el malestar urbano se canaliza desde propuestas de izquierda, mientras que en la Europa del Este y del Norte son populismos de derecha y nuevos partidos xenófobos quienes han canalizado sin la inseguridad y los crecientes sentimientos de repliegue.

Las causas que explican este difuso malestar son muchas. En primer lugar, la quiebra moral del sistema y la imposición de una única visión y solución del problema. La hegemonía del pensamiento neoliberal y la mayor parte de las medidas sugeridas e impuestas por organismos e instituciones para afrontar la crisis implican recortes de prestaciones y derechos, precariedad en el empleo e incertidumbre.

En segundo lugar, el descrédito de la política y la endogamia de los partidos políticos tradicionales. Más allá de consideraciones sobre la responsabilidad de los ciudadanos en una sociedad tan corporativa y fragmentada como la nuestra, lo cierto es que los partidos políticos ocupan el lugar más bajo en las encuestas de prestigio social en España.

En tercer lugar, la forma de entender la democracia. No está claro para muchos ciudadanos dónde está el poder, qué capacidad tienen hoy los Estados frente a los mercados y cómo la democracia representativa, a escala estatal, puede contrarrestar procesos globales que no conocen fronteras. La partitocracia tradicional reduce los espacios de participación, los partidos se apropian de los procesos, la democracia se empequeñece. Esto se traduce en decepción y desafección, pero no en desentendimiento. Por eso no son tanto movimientos contra el sistema o contra la democracia, sino precisamente lo contrario: reclaman un cambio radical, cambios políticos y más democracia. 


\section{CONCLUSIONES}

Los casos de Madrid y Valencia han sido un lugar de experimentación para desarrollar una agenda impulsada «desde arriba», un modelo al servicio de las elites, promotores inmobiliarios y determinados sectores de negocio. Es en las ciudades, en especial desde 2009, donde se han materializado las consecuencias de ese modelo y los efectos de la recesión y las políticas de recorte del gasto en servicios públicos fundamentales.

Nosotros creemos que es necesario y posible impulsar una nueva hoja de ruta en la formulación de políticas para la ciudad. La sugerente y muy completa agenda propuesta por Jordi Borja bien podría ser a nuestro juicio un excelente punto de partida (2012). Destacamos especialmente tres grandes ámbitos.

A la vista de investigaciones recientes (VVAA, 2013; Cucó, 2013a), los esfuerzos debieran orientarse, en primer lugar, a reducir la «fractura social». Tal vez sea el reto más difícil de afrontar (desempleo, procesos de fragmentación y reproducción segregada, gestión de la diversidad, atención a los grupos sociales de riesgo...) pero hoy es el más urgente y el que encierra mayor significado político. En rotunda afirmación de Oriol Nel·lo, que aquí se comparte, las ciudades han de hacer frente a tres retos fundamentales: la desigualdad, la desigualdad y la desigualdad (2015). La legitimidad de la acción de gobierno vendrá dada cada vez más por la vía de la igualdad y de la cohesión social. Con otro enfoque de políticas y a partir de un modelo de ciudad más inclusiva y sostenible, es imprescindible ocuparse del profundo impacto territorial de la recesión con prioridades claras: políticas locales de bienestar, cohesión social y equipamientos básicos (vivienda y atención a barrios degradados, tanto centrales como periféricos). Algunas buenas experiencias nos indican que hay que empezar por el barrio, la escuela y los servicios socio-sanitarios (VVAA, 2011; Nel.lo, 2011; Montaner y Subirats, 2012).

En segundo lugar, reducir la «brecha política» existente entre ciudadanos y partidos políticos. Es en las ciudades y regiones metropolitanas donde deben impulsarse innovaciones democráticas que vayan más allá de las formas tradicionales de democracia representativa o electoral, como única vía para combatir la creciente desafección democrática. Información, participación y consulta pública son los principios inspiradores, todavía en fase inicial, de esa nueva generación de iniciativas en materia de elaboración de presupuestos participados, e-democracy, e-transparency y e-participation que ya empiezan a ver la luz en algunas ciudades (Smith, 2009; Gutiérrez-Rubí, 2013; Borja, 2013).

Por último, impulsar modelos de crecimiento equilibrado y con visiones que tengan en cuenta la escala metropolitana. «Pensar juntos» en clave de región metropolitana es fundamental para «equipar» mejor a las ciudades y para introducir coherencia a las políticas públicas mejorando, con formas diversas y flexibles de gobierno del territorio, la coordinación y la cooperación entre actores políticos y entre actores públicos y privados.

\section{CODA}

Los resultados electorales del 24 de mayo de 2015 han supuesto un enorme cambio en el mapa municipal español. El modelo de políticas neoliberales para la ciudad ha sido mayoritariamente rechazado por los ciudadanos. Las elecciones locales han sido la suma de un inmenso capital político acumulado durante años en forma de movimientos sociales (mareas, plataformas, 
salvemos....) que constituían expresiones del malestar por los procesos de privatización de servicios públicos básicos, los recortes sociales y los episodios de corrupción política. Los ciudadanos hemos decidido situar en el centro de atención conceptos fundamentales como pacto, coordinación, cooperación, gobierno multinivel, transparencia, participación, inclusión, justicia social y ética pública. En definitiva, derecho a la ciudad, rescatar a las personas, mejor democracia.

En muchas grandes ciudades y en centenares de municipios los ciudadanos han otorgado la responsabilidad de gobernar a nuevas plataformas ciudadanas surgidas de movimientos sociales durante estos últimos años. Frente a la constatación, cada vez sentida por más gente en los años pasados, de que «no nos representan», las elecciones han permitido elegir a personas nuevas más en sintonía con las preocupaciones de la ciudadanía.

Los resultados electorales abren la posibilidad real de impulsar otro modelo de ciudad y una nueva agenda local para el cambio «desde abajo» (Romero y Boix, 2015). Un cambio que ha de poner nuestras Administraciones públicas al servicio de la ciudadanía. Para ello será necesario evaluar en toda su dimensión el creciente proceso de segmentación, precarización y aumento de las desigualdades en nuestras sociedades y analizar sus consecuencias políticas, sociales y culturales. Proponer algún tipo de alternativa para poblaciones mayoritariamente urbanas que expresan un grado de inseguridad, vulnerabilidad, incertidumbre y desconcierto respecto del futuro como nunca en las últimas décadas.

La nueva mayoría ha otorgado la alcaldía de Madrid a la plataforma ciudadana de unión popular «Ahora Madrid», que con el apoyo del Partido Socialista da fin a los 24 años de gobierno del Partido Popular. Se abre el escenario a cambios en el modelo de ciudad propuesto por el Plan General de Ordenación Urbana, elaborado por el gobierno saliente y sin tiempo para aprobarse antes de las elecciones. Dicho documento perpetuaba el modelo de desmantelamiento de la ciudad, el expolio de los suelos y edificios públicos para ponerlos al servicio de los intereses privados. El cambio se encuentra respaldado por colectivos que lo venían reclamando desde hace tiempo. Cabe citar la plataforma «No a este plan urbanístico», compuesta por asociaciones vecinales, ecologistas, de defensa del patrimonio, urbanistas, sindicatos y partidos políticos, así como el Colegio Oficial de Arquitectos de Madrid o la Federación Regional de Asociaciones de Vecinos de Madrid.

Uno de los problemas que la nueva corporación somete a revisión es el producido por la burbuja financiero inmobiliaria que ha hecho depender la política espacial y del suelo del impulso al desarrollo desordenado de suelo residencial hasta el límite del término municipal sobre territorios en su mayoría destinados anteriormente para anillo verde. Otra de las actuaciones que debería considerarse es la revisión integral de los planteamientos y objetivos de las grandes operaciones de reproducción de ciudad proyectadas en el interior, especialmente las de Chamartín, Mahou-Calderón y Campamento, por su dimensión y alcance. Son operaciones urbanísticoespeculativas que tienden a reforzar la desigualdad y que es preciso reconducir de manera que se adapten a las necesidades de la ciudad y sus plusvalías se orienten a la ciudadanía.

La ciudad de Valencia, cuyo gobierno local cuenta con una nueva mayoría integrada por la izquierda plural, también tendrá que saber encontrar espacios para impulsar otras políticas que den respuesta a los problemas concretos de sus conciudadanos. Haciendo posible que la ciudad como espacio político se construya desde abajo, encauzando nuevas formas de participación, dando sentido a la Valencia democrática, dejando «que las cosas buenas pasen» (Estal et al, 2014: 35). 
Aquí la agenda inmediata también es amplia: atender situaciones de emergencia social y de pobreza infantil, revisión del PGOU, remunicipalizar algunos servicios públicos, desarrollar una nueva política para el comercio local, encontrar una solución distinta y consensuada para el barrio de El Cabanyal, recuperar políticas de regeneración, revitalización y rehabilitación del centro histórico, impulsar un proyecto sostenible y respetuoso para el puerto y revisar toda la política fallida de Grandes Eventos, entre otras cuestiones.

La ciudad de Valencia debería también liderar una propuesta que garantice la protección de la Huerta y que establezca una nueva visión para el área metropolitana, dando mayor coherencia a cuatro ámbitos urgentes pero desatendidos durante décadas: promoción económica, gestión sostenible de recursos y política territorial, cohesión social e impulso de formas flexibles de coordinación y cooperación supramunicipal.

Los nuevos gobiernos constituidos navegan ahora con la fuerza del viento a favor. La fuerza que otorga la confianza depositada por millones de ciudadanos que reclaman otras políticas para la ciudad. Todos los estudios recientes evidencian el desarrollo de una nueva cultura política y cívica en las ciudades y municipios, en especial entre los más jóvenes. Sobre esa base, los gobiernos locales pueden ayudar a construir algo nuevo y mejor.

\section{REFERENCIAS}

ASOCIACIÓN ESTATAL DE DIRECTORAS Y GERENTES DE SERVICIOS SOCIALES (2015): Evolución 2011-2015 de los datos básicos de la gestión de la atención a la dependencia y costes y financiación del sistema por Comunidades Autónomas. Madrid, julio de 2015. Disponible en http://www.directoressociales.com/images/documentos/dictamenes/XV\%200\%20Dependencia\%20CC\%20AA\%20julio15.pdf

AYALA, L., MARTÍNEZ, R. y RUIZ-HUERTA, L. (2013): «Desigualdad y redistribución en los países de la OCDE» en Primer Informe sobre la Desigualdad en España. (Fundación Alternativas). Madrid, 26-72.

BECK, U. (2012): «La política económica de la inseguridad» en El País, 27 de mayo.

BORJA, J. (2010): Luces y sombras del modelo Barcelona. Barcelona. UOC.

BORJA, J. (2012): «El fin de la anticiudad postmodernista y el derecho a la ciudad en las regiones metropolitanas» en Ciudades, una ecuación imposible (Belil, M., et al, Eds.). Barcelona. Icaria, 279-320.

BORJA, J. (2013): Revolución urbana y derechos ciudadanos. Madrid. Alianza Editorial.

BRANDIS, D. y DEL RÍO, I. (2007): «Los últimos desarrollos urbanos en la periferia de la ciudad de Madrid» en Los procesos urbanos postfordistas (Artigues, A. et al, Eds.). Palma. AGE / Universitat de les Illes Balears, 71- 87.

BRANDIS, D. (2012a): «Los grandes desarrollos residenciales de la periferia de Madrid: de la burbuja a la crisis inmobiliaria» en Ciudades y paisajes en el siglo XXI (Delgado, C. et $a l$, Eds.). Santander. Estudio, 241-261.

BRANDIS, D. (2012b): «El estancamiento de los últimos desarrollos urbanos de la periferia madrileña. Crisis inmobiliaria y estrategias de ordenación» en Las ciudades y el sistema urbano. Reflexiones en tiempos de crisis (Miramontes, A. et al, Coords.). Santiago de Compostela. Meubook, 111-120. 
BRANDIS, D. (2014): «La producción inmobiliaria de la periferia madrileña (1991-2013)» en Desafíos metropolitanos (Michelini, J.J., Ed.). Madrid. La Catarata, 169-189.

BURRIEL, E. (2008): «La década prodigiosa del urbanismo español» en Scripta Nova, Vol. XII, no 270 (64). Universidad de Barcelona. Disponible en http://www.ub.es/geocrit/sn/ sn-199htm

BURRIEL, E. (2014): «El estallido de la burbuja inmobiliaria y sus efectos en el territorio» en Geografía de la crisis económica en España (Albertos, J.M. y Sánchez, J.L., Coords.). Valencia. PUV, 101-140.

CAMPOS, J.L. (2008): La burbuja inmobiliaria. Madrid. Marcial Pons.

CAPEL, H. (2005): El modelo de Barcelona. Un examen crítico. Barcelona. El Serbal.

CASTELLÓ, V. (2013): Los jóvenes más vulnerables frente al paro. Valencia. CES. Disponible en http://www.ces.gva.es/pdf/trabajos/revistas69/art2/pdf.

CASTELLS, M. (2011): «Situarse fuera del sistema político para obligarlo a cambiar» en $L a$ Vanguardia, 22 de octubre.

CIS Y MINISTERIO DE SANIDAD, SERVICIOS SOCIALES E IGUALDAD (2012): Barómetro Sanitario, 2012. Estudios núm. 2.396, 2.946 y 2.962, marzo-noviembre de 2012 (publicado el 14 de agosto de 2013).

CRUE (2015): La universidad española en cifras (2013-2014). Edición a cargo de J. Hernández y J. A. Pérez, Madrid.

CUCÓ, J. (Dir.) (2013a): La ciudad pervertida. Una mirada sobre la Valencia global. Madrid. Anthropos.

CUCÓ, J. (2013b): «Éxitos y perversiones en las fórmulas neoliberales. Los contrastes entre Barcelona, Bilbao y Valencia» en La ciudad pervertida. Una mirada sobre la Valencia global (Cucó, J., Dir.). Madrid. Anthropos, 213-244.

DAVIA, M.A. (2013): «Mercado de trabajo y desigualdad» en Primer Informe sobre la Desigualad en España. (Fundación Alternativas). Madrid, 75-133.

DELGADO, M. (2007): La ciudad mentirosa. Fraude y miseria del modelo Barcelona. Madrid. La Catarata.

DÍAZ, F. (2013): «Sociedad, espacio y crisis de la ciudad neoliberal» en Metamorfosis urbanas. Ciudades españolas en la dinámica global (Cucó, J., Ed.). Barcelona. Icaria, 81-107.

ESTEBAN, I. (2008): El efecto Guggenheim. Del espacio basura al ornamento. Barcelona. Anagrama.

ESTAL, D., MARRADES, R. y SEGOVIA, C. (2014): La Ciutat Construïda. Del Pla Urbanístic al Procés Ciutadà. Fundació Nexe. Disponible en http://www.fundacionexe.org/ documents/demos006.pdf.

FERNÁNDEZ DURÁN, R. (2008): «El Tsunami urbanizador español y mundial». Disponible en http://www.habitat.ag.upm.es/boletin/n38/arfer.html

FUNDACION FOESSA Y CÁRITAS (2012): Exclusión y Desarrollo Social. Madrid. Fundación FOESSA y Cáritas Española.

GAJA, F. (2013): «Tras el tsunami inmobiliario. Salir del atolladero» en Paisajes devastados después del ciclo inmobiliario: impactos regionales y urbanos de la crisis (Observatorio Metropolitano). Madrid. Traficantes de Sueños, 313-353.

GRADIN, C. y DEL RÍO, C. (2013): «El desempleo de inmigrantes, mujeres y jóvenes» en Primer Informe sobre la Desigualad en España (Fundación Alternativas). Madrid, 136-191. 
GUTIÉRREZ-RUBÍ, A. (2013): «Queremos legislar», en El País, 24 de marzo.

HARVEY, D. (2012): «Las raíces urbanas de las crisis financieras: reclamar la ciudad para la lucha anticapitalista» en Ciudades, una ecuación imposible (Belil, M. et al, Eds.). Barcelona, Icaria, 321-358.

HERNÁNDEZ, G.M. y TORRES, F. (2013): «El impacto de la Valencia glocalizada en el Centro Histórico popular», en La ciudad pervertida. Una mirada sobre la Valencia global. (Cucó, J. Ed.). Madrid. Anthropos, 19-40.

HERRERO, C., SOLER, A. y VILLAR, A. (2012): La pobreza en España y sus Comunidades Autónomas. Valencia. IVIE.

KRUGMAN, P. (2008): Después de Bush. El fin de los «neocons» y la hora de los demócratas. Barcelona. Crítica.

JÁTIVA, J.M. (2013): «Catalá impulsa una revolución conservadora con las privatizaciones» en El País, 2 de septiembre.

MÉNDEZ, R. (2012): «Globalización, neoliberalismo y dinámicas metropolitanas en Madrid» en DAAPGE, vol. 12, n 19, 29-49, Santa Fe (Argentina). Disponible en http:// www.scielo.org.ar/

MÉNDEZ, R., ABAD, L. y ECHAVES, C. (2015): Atlas de la crisis. Valencia. Tirant Lo Blanch

MINISTERIO DE HACIENDA Y ADMINISTRACIONES PÚBLICAS (2013): Actualización del Programa de Estabilidad del Reino de España 2013-2016, 26 de abril.

MONTANER, J.M. y SUBIRATS, J. (Eds.) (2012): Repensar las políticas urbanas. Barcelona. Diputación de Barcelona.

MORENO, L. (2013): La Europa asocial. Madrid. Península.

NAREDO, J.M. (2009): «La cara oculta de la crisis. El fin del boom inmobiliario y sus consecuencias» en Revista de Economía Crítica, n ${ }^{\circ}$ 7, 313-340.

NAREDO, J.M. (2010): «El modelo inmobiliario español y sus consecuencias» en Ciudades para un futuro más sostenible. Disponible en http://habitat.aq.upm.es

NAREDO, J.M. y MONTIEL MÁRQUEZ, A. (2011): El modelo inmobiliario español y su culminación en el caso valenciano. Barcelona. Icaria.

NEL.LO, O. (2011): «The five challenges of urban rehabilitation. The Catalan experience» en Urban Research and Practice, vol. 4, $\mathrm{n}^{\circ}$ 3, 308-325.

NEL·LO, O. (2015): «Barcelona: un pla per als barris contra la desigualtat». Disponible en http://oriolnello.blogspot.com.es/2015/04/barcelona-un-pla-per-als-barris-contra.html

OBSERVATORIO DE LA DEPENDENCIA (2013): X Dictamen del Observatorio de la Dependencia. Madrid.

PINSON, G. (2011): Urbanismo y gobernanza de las ciudades europeas. Gobernar la ciudad por proyecto. Valencia, Universidad de Valencia.

PITARCH, M.D. (2014): «Desigualdades regionales, pobreza y vulnerabilidad social en España durante la crisis (2007-2013)» en Geografía de la crisis económica en España (Albertos, J.M. y Sánchez, J.L., Coords.). Valencia, Publicacions de la Universitat de València, 201-229.

RODRÍGUEZ, E., GARCÍA, B. y MUÑOZ, O. (2013): «Del Madrid global a la crisis urbana» en Paisajes devastados después del ciclo inmobiliario: impactos regionales y urbanos de la crisis (Observatorio Metropolitano). Madrid. Traficantes de Sueños, 123-177. 
ROMERO, J., JIMÉNEZ, F. y VILLORIA, M. (2012): «(Un)sustainable territories: causes of the speculative bubble in Spain (1996-2010) and its territorial, environmental, and sociopolitical consequences» en Environment and Planning C: Government and Policy, vol. 30, n $3,467-486$.

ROMERO, J. (2013): «De nuevo la Cuestión Social en Europa. Una visión alternativa a la del pensamiento conservador y agenda para la investigación» en Scripta Nova, vol. XVII, $\mathrm{n}^{\circ}$ 444. Universidad de Barcelona, 10 de julio de 2013. Disponible en http://www.ub.es/ geocrit/sn/sn-444.htm

ROMERO, J. y BOIX, A. (Eds.) (2015): Democracia desde abajo. Nueva agenda para los gobiernos locales. Valencia, Publicacions de la Universitat de València. .

ROMERO, J., COLLADO, J.C. y RODRÍGUEZ, M.T. (2014): «La economía política de la desigualdad. Los recortes en el sector público y sus repercusiones para el Estado de Bienestar en España» en Geografía de la crisis económica en España (Albertos, J.M. y Sánchez, J.L., Coords.). Valencia. PUV, 329-376.

ROMERO, J. y FARINÓS, J. (2013): «Cities and Urban and Metropolitan Regions in Spain: A New Agenda in a Global Context» en Urban Governance in Southern Europe (Seixas, J. y Albert, A., Eds.). Farnham. Ashgate, 123-148.

ROMERO, J. y FRANCÉS, M. (Eds.) (2012): La Huerta de Valencia. Un paisaje cultural con futuro incierto. Valencia, Publicacions de la Universitat de València..

ROMERO, J. y MELO, C. (2015): «Spanish Mediterranean Huertas: theory and reality in the planning and management of peri-urban agriculture and cultural landscapes» en WIT Transactions on Ecology and the Environment, $\mathrm{n}^{\circ} .193,585-595$.

ROSANVALLON, P. (2012): La sociedad de los iguales. Barcelona. RBA.

RUIZ, M.A. y SANTAMARINA, B. (2013): «La Valencia bipolar y trepidante. Discursos y representaciones sobre transformación urbana» en La ciudad pervertida. Una mirada sobre la Valencia global (Cucó, J., Ed.). Madrid. Anthropos, 117-140.

SALOM, J. y ALBERTOS, J.M. (2014): «La crisis económica en los distritos industriales valencianos» en Geografía de la crisis económica en España (Albertos, J.M. y Sánchez, J.L., Coords.). Valencia. Publicacions de la Universitat de València, 467-495.

SAVAGE, M. et al (2013): «A New Model of Social Class? Findings from the BBC's Great British Class Survey Experiment» en Sociology, 47 (2), pp. 219-250.

SAVE THE CHILDREN (2013): 2.826.549 razones. La protección de la infancia frente a la pobreza: un derecho, una obligación y una inversión. Disponible en http://www.savethechildren.es/docs/Ficheros/644/INFORME.pdf.

SCHRAAD-TISCHLER, D. y KROLL, C. (2014): Social Justice in the EU. A Cross-national Comparison. Social Inclusion Monitor Europe (SIM)-Index Report Bertelsmann Stiftung. Disponible en https://www.bertelsmann-stiftung.de/en/publications/publications/ publication/did/social-justice-in-the-eu-a-cross-national-comparison/

SMITH, G. (2009): Democratic innovations. Cambridge. Cambridge University Press.

SORRIBES, J. (2015): Valencia, 1940-2014. Construcción y destrucción de la ciudad. Valencia. Publicacions de la Universitat de València.

STANDING, G. (2013): El precariado. Una nueva clase social. Barcelona. Pasado y Presente.

STANNERS, D. \& BOURDEAU, P. (eds.) (1995): Europe's environment: The Dobris assessment. Copenhagen. European Environmental Agency. 
TORRES, F. y GARCÍA, P. (2013): «La ciudad ocultada. Desigualdad y precarización en la Valencia global» en La ciudad pervertida. Una mirada sobre la Valencia global (Cucó, J. Ed.). Madrid. Anthropos, 163-188.

UNICEF (2014): La infancia en España 2014. El valor social de los niños: hacia un Pacto de Estado por la infancia. Madrid. Junio de 2014. Disponible en http://www.unicef.es/ sites/www.unicef.es/files/infancia-espana/unicef_informe_la_infancia_en_espana_2014. pdf.

VINUESA, J. (2013): El festín de la vivienda. Auge y caída del negocio inmobiliario en España. Madrid. Díaz \& Pons.

VIVES, S. y RULLÁN, O. (2014): «La apropiación de las rentas del suelo en la ciudad neoliberal española» en Boletín de la Asociación de Geógrafos Españoles, nº 65, 387-408.

VVAA (2011): Arquitectura, Ciudad y Entorno, n 17, (Número monográfico sobre ciudades centrales en Europa). Barcelona, Universidad Politécnica de Cataluña.

OBSERVATORIO METROPOLITANO (2013): Paisajes devastados después del ciclo inmobiliario: impactos regionales y urbanos de la crisis. Madrid. Traficantes de Sueños.

ZORNOZA, C. (2013): Crecimiento urbanístico en la zona costera de la Comunidad Valenciana (1987-2009). Análisis y perspectivas de futuro. Valencia. Publicacions de la Universitat de València. 